\title{
P 062 NO SUCH THING AS BAD PUBLICITY? QUALITATIVE STUDY OF STUDENTS AND DOCTORS LEARNING NEEDS FOR END-OF-LIFE CARE IN THE MEDIA LIMELIGHT
}

Henrietta Edge, Sarah Yardley. Keele University, Keele, UK

10.1136/bmjspcare-2014-000654.103

Background Death and dying are an unavoidable part of medical practice but doctors continue to report lack of competence in end-of-life care. Negative news media has placed additional demands on doctors seeking to negotiate issues in end-of-life care.

Aims This study addresses three questions

1. What is the awareness among final year students and newly qualified doctors of media attention to end-of-life care?

2. Do these groups expect media attention to affect practice with respect to end-of-life care?

3. What are the self-identified learning needs of these groups with respect to providing end-of-life care and responding to media attention?

Methods This study draws on experiential learning theories, recognizing that learning of 'good medical practice' is influenced by context. Following a literature review we undertook a thematic analysis of coverage of the Liverpool Care Pathway in three purposively selected national newspapers. Volunteer students $(n=8)$ and newly qualified doctors $(n=8)$ are being recruited to participate in two sets of longitudinal focus groups. The first session explores research questions $1 / 2$. The next introduces our media analysis to extend discussions. In the final session we will discuss the emerging analysis and specifically explore perceptions of learning needs. Data will be audio-recorded and transcribed verbatim. An iterative approach to qualitative thematic analysis concurrent with data generation will be used to describe and interpret the overall findings.

Results We will present our findings combining data from media analysis with longitudinal focus groups. Implications including the identification of learning needs with respect to end-of-life care and professional duties when responding to media attention will be discussed.

Conclusions External influences on medical practice, such as media attention, can influence understandings of a phenomenon, challenge or legitimize particular practices. This study provides important insights into perceptions and learning needs of students and newly qualified doctors regarding end-of-life care. 